Pure and Applied Mathematics Quarterly

Volume 5, Number 1

(Special Issue: In honor of

Jean-Pierre Serre, Part 2 of 2$)$

$469-494,2009$

\title{
Characteristic $p$ Analogue of Modules with Finite Crystalline Height
}

\author{
Victor Abrashkin ${ }^{1}$
}

\begin{abstract}
In the case of local fields of positive characteristic we introduce an analogue of Fontaine's concept of Galois modules with crystalline height $h \in \mathbb{N}$. If $h=1$ these modules appear as geometric points of Faltings's strict modules. We obtain upper estimates for the largest upper ramification numbers of these modules and prove (under an additional assumption) that these estimates are sharp.
\end{abstract}

Keywords: local fields, ramification filtration, crystalline representations, Anderson motives

\section{Introduction.}

Let $p$ be a prime number. Let $K$ be a complete discrete valuation field with perfect residue field $k$ of characteristic $p$. Choose a separable closure $K_{\text {sep }}$ of $K$ and set $\Gamma_{K}=\operatorname{Gal}\left(K_{\mathrm{sep}} / K\right)$. Denote by $R$ the valuation ring of $K$ and for any $v>0$, by $\Gamma_{K}^{(v)}$ the ramification subgroup of $\Gamma_{K}$ with the upper number $v$.

Suppose, first, that $K$ is of characteristic 0, i.e. $K$ contains $\mathbb{Q}_{p}$, and consider $e=e(K)$ - the ramification index of $K$ over $\mathbb{Q}_{p}$. In this situation for $h \in \mathbb{N}$, Fontaine [Fo3] introduced the category $\mathrm{MG}_{K}^{h}$ of finite $\mathbb{Z}_{p}\left[\Gamma_{K}\right]$-modules with crystalline height $h$. Examples of such modules are given by subquotients of crystalline representations of $\Gamma_{K}$ with the Hodge-Tate filtration of length $h$ or, more

Received August 8, 2007.

1991 Mathematics Subject Classification. 11S15, 11S20.

$1_{\text {Partially supported by EPSRC, GR/S72252/01 }}$ 
specifically, of Galois modules of $h$-th etale cohomology of projective schemes over $K$ with good reduction. If $h=1$ then the corresponding Galois modules appear as points $\mathcal{G}\left(K_{\mathrm{sep}}\right)$ of finite flat $p$-group schemes (i.e. group schemes killed by a power of the endomorphism $\left.p \operatorname{id}_{\mathcal{G}}\right) \mathcal{G}$ over $R$. In this case Fontaine [Fo1] proved a very important ramification estimate:

( $\alpha)$ if $G \in \mathrm{MG}_{K}^{1}, p^{N} G=0$ and $v>e\left(N+\frac{1}{p-1}\right)-1$ then the ramification subgroup $\Gamma_{K}^{(v)}$ acts trivially on $G$.

This result was generalised in [Ab2] (cf. also [Fo2], [Ab1,3]):

$(\beta)$ if $G$ is a subquotient of crystalline representation of $\Gamma_{K}$ with the Hodge-Tate filtration of length $h<p-1, p^{N} G=0$ and $e=1$ then for $v>\left(N+\frac{h}{p-1}\right)-1$, $\Gamma_{K}^{(v)}$ acts trivially on $H$.

Now suppose that $K$ is of characteristic $p$ and $k \supset \mathbb{F}_{q}$, where $q$ is a power of $p$. Introduce an analogue of $\mathbb{Z}_{p}$. This will be a subring $O=\mathbb{F}_{q}[[\pi]]$ of $R$, where $\pi \in R$ is not invertible in $R$. If $K_{0}$ is the fraction field of $O$ in $K$ then denote by $e=e\left(K / K_{0}\right)$ the ramification index of $K$ over $K_{0}$. In this situation an analogue of the category of finite flat $p$-group schemes over $R$ is the category of $O$-strict modules over $R$ with etale generic fibre. (The concept of $O$-strict module was introduced in $[\mathrm{Fa}]$.$) This category was studied in details in [\mathrm{Ab6}]$, where the following ramification estimate ${ }^{2}$ was obtained:

$(\gamma)$ if $G=\mathcal{G}\left(K_{\mathrm{sep}}\right)$, where $\mathcal{G}$ is an $O$-strict module over $R$ and $\pi^{N} G=0$ then for $v>e\left(N+\frac{1}{q-1}\right)-1, \Gamma_{K}^{(v)}$ acts trivially on $G$.

This estimate is a complete analogue of the above Fontaine's estimate for $p$ group schemes in the mixed characteristic case. Notice that all $O$-strict modules appear as kernels of isogenies of Drinfeld modules, cf. [Ab6]. The above result gives another evidence that Galois modules arising from torsion points of Drinfeld modules give a perfect analogue of first etale cohomology in the mixed characteristic case. If we try to think about equicharacteristic interpretation of

$\overline{2 \text { the statement }}$ of this result in the Introduction to $[\mathrm{Ab} 6]$ contains a misprint 
higher etale cohomology it looks natural to consider the Galois modules arising from Anderson's motives, cf. eg. [An] for their definition. This idea perfectly matches with Fontaine's definition of Galois modules of finite crystalline height $h$ in the mixed characteristic case. Let $\mathrm{MG}^{h}(O)_{K}$ be the corresponding category of $O\left[\Gamma_{K}\right]$-modules of "finite crystalline height" $h \in \mathbb{N}$ (it is defined in Section 1). Notice that if $G \in \mathrm{MG}^{1}(O)_{K}$ then $G$ appears in the form $\mathcal{G}\left(K_{\text {sep }}\right)$, where $\mathcal{G}$ is $O$-strict module over $R$. In section 3 we apply methods from [Ab6] to prove the following ramification estimate:

( $\delta)$ if $G \in \mathrm{MG}^{h}(O)_{K}$ and $\pi^{N} G=0$ then for $v>e\left(N-1+\frac{h q}{q-1}\right)-1, \Gamma_{K}^{(v)}$ acts trivially on $G$.

In section 4 it is shown that this ramification estimate can not be improved under the sufficiently general assumption $\left(\begin{array}{c}-h \\ N-1\end{array}\right) \not \equiv 0 \bmod p$. In particular, the estimate $(\delta)$ does not match with the above mentioned estimate for subquotients of crystalline representations in the mixed characteristic case. One can say that in the case of local fields of positive characteristic the Galois modules with finite crystalline height do not give a precise analogue of crystalline representations. Notice that the above ramification estimates $(\alpha)$ and $(\beta)$ constitute an essential ingredient of the proof of the Shafarevich Conjecture and its generalizations, $[\mathrm{Fo} 1,2],[\mathrm{Ab} 1,3],[\mathrm{Sc}]$. In the equicharacteristic case there are no such interesting applications of the corresponding estimates $(\gamma)$ and $(\delta)$. But anyway these estimates give a non-trivial information about torsion points of Anderson motives and also some ideas what one can expect when studying crystalline representations in the mixed characteristic case.

The proof of estimate $(\delta)$ uses essentially the existence of embedding of any $G \in$ $\mathrm{MG}^{h}(O)_{K}$ in a $\pi$-divisible group consisting of objects of the category $\mathrm{MG}^{h}(O)_{K}$. This statement is parallel to the corresponding statement for $h=1$ from [Ab6] and is proved in section 2. Due to this result we can assume that $G$ is a free $O / \pi^{N}$-module, this allows us to have much better control on equations of the $K$-scheme $\mathcal{G}$ such that $\mathcal{G}\left(K_{\text {sep }}\right)=G$.

The method of proving the ramification estimates from sections 3 and 4 can be used only in the characteristic $p$ case. It was applied earlier by the author, cf. eg. [Ab4,5]. The basic idea of this method can be explained as follows. 
Suppose $\alpha>0, \alpha \in \mathbb{Q}$ and its $p$-adic valuation is zero. Then there is a finite totally ramified extension $K_{\alpha}$ of $K$ such that its Herbrand function has a unique edge point $(\alpha, \alpha)$, cf. eg. [Ab4, n.1.5]. Consider a natural embedding $\iota: \Gamma_{K_{\alpha}}=\operatorname{Gal}\left(K_{\mathrm{sep}} / K_{\alpha}\right) \longrightarrow \Gamma_{K}$ and the corresponding $\Gamma_{K_{\alpha}}$-module $\iota^{*}(G)$. Let $\iota_{\alpha}: \Gamma_{K_{\alpha}} \longrightarrow \Gamma_{K}$ be an isomorphism of profinite groups induced by an isomorphism of local fields $K$ and $K_{\alpha}$ (they both have the same residue field and, therefore, are isomorphic). Introduce the corresponding $\Gamma_{K_{\alpha}}$-module $\iota_{\alpha}^{*}(G)$. Then

if points of $\iota^{*}(G)$ and $\iota_{\alpha}^{*}(G)$ have the same common field of definition over $K_{\alpha}$ then $\Gamma_{K}^{(v)}$ acts trivially on $G$ for all $v>\alpha$.

This fact follows easily from elementary properties of Herbrand functions and is proved in a slightly more general form in n.3.1. Notation. Everywhere in the paper if $f: A \longrightarrow B$ and $g: B \longrightarrow C$ are morphisms then their composition will be denoted as $f g$, i.e. for any $a \in A,(f g)(a)=g(f(a))$.

Acknowledgement. The author expresses a deep gratitude to the referee for very careful reading of the original version of this paper. His critical remarks resulted in a considerable improvement of the quality of the exposition.

\section{Main notation and results}

\subsection{The categories $\bmod (O)_{R}$ and $\mathrm{MG}(O)_{K}$}

As in the introduction, let $q \in \mathbb{N}$ be a power of a prime number $p$. Let $O=\mathbb{F}_{q}[[\pi]]$ be a ring of formal power series in one fixed indeterminate $\pi$ and denote by $K_{0}$ the fraction field of $O$. Let $R$ be an $O$-algebra. Everywhere in the paper $R$ is an integral domain with fraction field $K$. (In sections 3 and $4 K$ will be a complete discrete valuation field.) Choose a separable closure $K_{\text {sep }}$ of $K$ and set $\Gamma_{K}=\operatorname{Gal}\left(K_{\mathrm{sep}} / K\right)$. Denote by $\sigma=\sigma_{q}: R \longrightarrow R$ the ring endomorphism of $R$ such that $\sigma(r)=r^{q}$ for any $r \in R$.

Let $\operatorname{MG}(O)_{K}$ be the category of $O$-modules of finite type with continuous $O$-linear action of $\Gamma_{K}$.

Introduce the category $\bmod (O)_{R}$ of triples $(L, F,[\pi])$, where 
- $L$ is a free $R$-module of finite rank;

- if $L^{(q)}=L \otimes_{(R, \sigma)} R$ then $F: L^{(q)} \longrightarrow L$ is an injective $R$-linear morphism;

- $[\pi] \in \operatorname{End}_{R} L$ is nilpotent and $F[\pi]=[\pi]^{(q)} F$, where $[\pi]^{(q)}:=[\pi] \otimes$ id $\epsilon$ $\operatorname{End}_{R}\left(L^{(q)}\right)$.

If $\mathcal{L}=(L, F,[\pi])$ and $\mathcal{L}_{1}=\left(L_{1}, F,[\pi]\right)$ are two objects from $\bmod (O)_{R}$ then Hom $\bmod (O)_{R}\left(\mathcal{L}, \mathcal{L}_{1}\right)$ consists of $R$-linear morphisms $f: L \longrightarrow L_{1}$ such that $f^{(q)} F=F f$ and $f[\pi]=[\pi] f$.

Remark. We have a natural embedding $\operatorname{End}_{R} L \subset \operatorname{End}_{K}\left(L \otimes_{R} K\right)$. Therefore, if $\mathrm{rk}_{R} L=s$ then $[\pi]^{s}=0$.

\subsection{Functor $\mathcal{M}_{\Gamma}: \bmod (O)_{R} \longrightarrow \operatorname{MG}(O)_{K}$}

Let $\mathcal{L}=(L, F,[\pi])$ be an object of the category $\bmod (O)_{R}$. Consider the $R$ algebra $A=A(\mathcal{L}):=\left(\operatorname{Sym}_{R} L\right) / I$, where the ideal $I$ is generated by the elements $l^{q}-F(l \otimes 1) \in \operatorname{Sym}_{R} L$ for all $l \in L$. Because $F$ is injective, for $A_{K}=A \otimes_{R} K$, we have $\Omega_{A / K}^{1}=0, A_{K}$ is an etale $K$-algebra and $\operatorname{rk}_{R} A=\operatorname{dim}_{K} A_{K}=q^{\mathrm{rk}_{R} L}$. In particular, if $\mathcal{G}=\operatorname{Spec} A$ then $\mathcal{G}\left(K_{\mathrm{sep}}\right)=\operatorname{Hom}_{R \text {-alg }}\left(A, K_{\mathrm{sep}}\right)$ consists of $q^{\mathrm{rk}_{R} L}$ elements. Notice that $\mathcal{G}$ has a natural structure of a group scheme over $R$ given by the comultiplication $\Delta_{A}: A \longrightarrow A \otimes_{K} A$ and the counit $e_{A}: A \longrightarrow K$ such that $\Delta_{A}(l)=l \otimes 1+1 \otimes l$ and $e_{A}(l)=0$ for all $l \in L$. Set $[\alpha](l)=\alpha l$ for $\alpha \in \mathbb{F}_{q}$ and $l \in L$. Introduce $[\pi]_{A}: A \longrightarrow A$, which is induced by the given above $[\pi] \in \operatorname{End}_{R} L$. As a result, we obtain a structure of $O$-comodule on $A$. Therefore, $\mathcal{G}\left(K_{\mathrm{sep}}\right)$ is an $O$-module with a natural continuous action of the Galois group $\Gamma_{K}$ i.e. $\mathcal{G}\left(K_{\mathrm{sep}}\right) \in \operatorname{MG}(O)_{K}$.

Clearly, the correspondence $\mathcal{L} \mapsto \mathcal{G}\left(K_{\text {sep }}\right)$ determines a functor $\mathcal{M}_{\Gamma}$ from $\bmod (O)_{R}$ to $\operatorname{MG}(O)_{K}$. As a matter of fact, with the above notation the correspondence $\mathcal{L} \mapsto \mathcal{G}$ induces antiequivalence of the category $\bmod (O)_{R}$ and the category of finite flat $p$-group schemes $\mathcal{G}$ over $R$ with etale generic fibre, zero Verschiebung $V_{G}$ and a structure of $O$-module scheme. For the proof in the case $O=\mathbb{Z}_{p}$, cf. [Ga]; the general case can be considered similarly.

1.3. The categories $\bmod ^{h}(O)_{R}$ and $\mathrm{MG}^{h}(O)_{K}$ 
Let $h \in \mathbb{N}$. Introduce the category $\bmod ^{h}(O)_{R}$ as a full subcategory in $\bmod (O)_{R}$ consisted of $\mathcal{L}=(L, F,[\pi])$ such that $\left(\pi \operatorname{id}_{L}-[\pi]\right)^{h}(L) \subset \operatorname{Im} F$.

Denote by $\mathrm{MG}^{h}(O)_{K}$ the full subcategory in $\operatorname{MG}(O)_{K}$ consisting of $O\left[\Gamma_{K}\right]$ modules $\mathcal{M}_{\Gamma}(\mathcal{L})$, where $\mathcal{L}$ is an object of the category $\bmod ^{h}(O)_{R}$.

We are going to prove the following three results:

- if $G \in \mathrm{MG}^{h}(O)_{K}$ then $G$ can be embedded into a $\pi$-divisible group of finite height, consisting of objects of the category $\mathrm{MG}^{h}(O)_{K}$;

- if $K$ is a complete dicrete valuation ring with perfect residue field, $e=$ $e\left(K / K_{0}\right), G \in \mathrm{MG}^{h}(O)_{K}$ and $\pi^{N} G=0$ then the ramification subgroups $\Gamma_{K}^{(v)}$ act trivially on $G$ for $v>e\left(N-1+\frac{q h}{q-1}\right)-1$;

- with the above notation and assumptions if $\left(\begin{array}{c}-h \\ N-1\end{array}\right) \not \equiv 0 \bmod p$ then the above ramification estimate is sharp.

Remark. In the context of classical $p$-group schemes an analogue of the above first result is Raynaud's theorem stating that any finite flat group scheme admits embedding into a $p$-divisible group (even into an abelian scheme). In the context of $O$-strict modules (the case $h=1$ ) this result was proved in [Ab6]. The case of arbitrary $h$ will be proved in the next section by esssentially the same method. It seems our method can be also applied to prove an analogue of this statement for Fontaine's modules of finite cryatalline height in the mixed characteristic case.

\section{Embedding into a $\pi$-divisible group.}

2.1. The concept of $\pi$-divisible group.

Tate's definition of $p$-divisible groups in the category of finite flat $p$-group schemes admits the following interpretation in the categories $\mathrm{MG}^{h}(O)_{K}$ and $\bmod { }^{h}(O)_{R}$.

A $\pi$-divisible group in the category $\operatorname{MG}^{h}(O)_{K}$ is an inductive system $\left\{G_{n}, i_{n}\right\}_{n \geqslant 1}$, where for any $n \in \mathbb{N}, G_{n} \in \mathrm{MG}^{h}(O)_{K}$ and $i_{n}: G_{n} \longrightarrow G_{n+1}$ are embeddings of $O\left[\Gamma_{K}\right]$-modules such that if $n>m$ and $i_{m n}: G_{m} \longrightarrow G_{n}$ is the composition of $i_{m}, \ldots, i_{n-1}$, then we have the short exact sequence

$$
0 \longrightarrow G_{m} \stackrel{i_{m n}}{\longrightarrow} G_{n} \stackrel{j_{n m}}{\longrightarrow} G_{n-m} \longrightarrow 0
$$


Characteristic $p$ Analogue of Modules with Finite Crystalline Height 475

and $j_{n m} i_{n-m, n}=\pi^{m} \operatorname{id}_{G_{n}}$.

The above definition can be also adjusted to the category $\bmod ^{h}(O)_{R}$ by introducing the concept of strict embedding. If $\mathcal{L}=(L, F,[\pi])$ and $\mathcal{L}_{1}=\left(L_{1}, F,[\pi]\right)$ then $i \in \operatorname{Hom} \bmod (O)_{R}\left(\mathcal{L}_{1}, \mathcal{L}\right)$ is a strict embedding if it is induced by $i: L_{1} \longrightarrow L$ such that $L / i\left(L_{1}\right)$ has no $R$-torsion. Such $i$ gives rise to a natural short exact sequence $0 \longrightarrow \mathcal{L}_{1} \longrightarrow \mathcal{L} \longrightarrow \mathcal{L}_{2} \longrightarrow 0$ in the category $\bmod (O)_{R}$. Then we can proceed similarly to introduce a $[\pi]$-divisible group as an inductive system $\left\{\mathcal{L}_{n}, i_{n}\right\}_{n} \geqslant 1$ of objects of the category $\bmod ^{h}(O)_{R}$, where all $i_{n}$ are strict embeddings.

\subsection{The statement of the first main theorem.}

Theorem A. If $G \in \mathrm{MG}^{h}(O)_{K}$ then there is a $\pi$-divisible group $\left\{G_{n}, i_{n}\right\}_{n \geqslant 1}$ in the category $\mathrm{MG}^{h}(O)_{K}$ such that if $N \in \mathbb{N}$ is such that $\pi^{N} \mathrm{id}_{G}=0$ then there is an embedding of $G$ into $G_{N}$ in the category $\operatorname{MG}(O)_{K}$.

The above theorem is implied by the following theorem.

Theorem A'. If $\mathcal{L} \in \bmod ^{h}(O)_{R}$ then there is a $[\pi]$-divisible group $\left\{\mathcal{L}_{n}, i_{n}\right\}_{n} \geqslant 1$ in $\bmod ^{h}(O)_{R}$ such that if $N \in \mathbb{N}$ is such that $\left[\pi^{N}\right] \mathcal{L}=0$ then there is an epimorphic map from $\mathcal{L}_{N}$ to $\mathcal{L}$ in the category $\bmod (O)_{R}$.

The proof of theorem $A^{\prime}$ will be given in nn.2.3-2.6 below.

Remark When introducing the category $\bmod ^{h}(O)_{R}$ one could start with $O=$ $\mathbb{F}_{q}[\pi]$ and consider any $O$-algebra $R$. Then essentially same arguments give an analogue of Theorem $A^{\prime}$ everywhere locally on $R$.

2.3. Suppose $\mathcal{L}=(L, F,[\pi]) \in \bmod { }^{h}(O)_{R}$.

Lemma 1. There is a unique $R$-linear $V=V_{\mathcal{L}}: L \longrightarrow L^{(q)}$ such that

a) $V[\pi]^{(q)}=[\pi] V$;

b) $V F=\left(\pi \operatorname{id}_{L}-[\pi]\right)^{h}$;

c) $F V=\left(\pi \operatorname{id}_{L^{(q)}}-[\pi]^{(q)}\right)^{h}$.

Proof. Because $F$ is injective and $\operatorname{Im} F \supset \operatorname{Im}\left(\pi \mathrm{id}_{L}-[\pi]\right)^{h}$, there is a unique $R$ linear $V$ such that $V F=\left(\pi \operatorname{id}_{L}-[\pi]\right)^{h}$. Then $V[\pi]^{(q)} F=V F[\pi]=[\pi] V F$ implies that $V[\pi]^{(q)}=[\pi] V$, because $F$ is injective. Similarly,

$$
F V F=F\left(\pi \operatorname{id}_{L}-[\pi]\right)^{h}=\left(\pi \operatorname{id}_{L^{(q)}}-[\pi]^{(q)}\right)^{h} F
$$


implies the part c) of our lemma.

\subsection{Matrix identities}

Suppose $\mathcal{L}=(L, F,[\pi])$ is an object of the category $\bmod ^{h}(O)_{R}$ and $V=V_{\mathcal{L}}$ is the morphism from n.2.3. Choose an $R$-basis $\bar{e}=\left\{e_{b}\right\}_{1 \leqslant b \leqslant s}$ of $L$ and consider square matrices $C=\left(c_{a b}\right), D=\left(d_{a b}\right), \Pi=\left(\gamma_{a b}\right) \in M_{s}(R)$ such that for $1 \leqslant b \leqslant s$,

$$
V\left(e_{b}\right)=\sum_{a} e_{a} \otimes c_{a b}, F\left(e_{b} \otimes 1\right)=\sum_{a} e_{a} d_{a b},[\pi]\left(e_{b}\right)=\sum_{a} e_{a} \gamma_{a b} .
$$

Then in obvious notation it holds $V(\bar{e})=\bar{e} \otimes C, F(\bar{e} \otimes 1)=\bar{e} D,[\pi](\bar{e})=\bar{e} \Pi$ and there are the following rules of composition

$$
\begin{gathered}
V F: \bar{e} \stackrel{V}{\longrightarrow} \bar{e} \otimes C \stackrel{F}{\longrightarrow} \bar{e} D C \\
F V: \bar{e} \otimes 1 \stackrel{F}{\longrightarrow} \bar{e} D \stackrel{V}{\longrightarrow} \bar{e} \otimes C D .
\end{gathered}
$$

The proof of the following proposition is quite straightforward.

Proposition 2. Suppose $\bar{e}=\left(e_{b}\right)_{1 \leqslant b \leqslant s}$ is a basis of a free $R$-module $L$ and $D=$ $\left(d_{a b}\right), \Pi=\left(\gamma_{a b}\right) \in M_{s}(R)$. Suppose $F: L^{(q)} \longrightarrow L$ is given by the correspondence $\bar{e} \otimes 1 \mapsto \bar{e} D$ and $[\pi]: L \longrightarrow L$ is given via $\bar{e} \mapsto \bar{e} \Pi$. Then $\mathcal{L}=(L, F,[\pi]) \in$ $\bmod { }^{h}(O)_{R}$ if and only if

(1) $\Pi D=D \Pi^{(q)}$, where $\Pi^{(q)}=\left(\gamma_{a b}^{q}\right)$;

(2) $\operatorname{det} D \neq 0$;

(3) $C:=D^{-1}\left(\pi I_{s}-\Pi\right)^{h} \in M_{s}(R)$, where $I_{s}$ is the identity matrix of size $s$;

(4) $\Pi$ is nilpotent.

Remark a) If above conditions (1)-(3) hold then $V: \bar{e} \mapsto \bar{e} \otimes C, C D=\left(\pi I_{s}-\Pi^{(q)}\right)^{h}$ and $\Pi^{(q)} C=C \Pi$.

b) Because $\Pi$ is nilpotent, $\operatorname{det}\left(\pi I_{s}-\Pi\right) \neq 0$ and, therefore, $\operatorname{det} C \neq 0$.

2.5. Construction of a $\pi$-divisible group in $\bmod ^{h}(O)_{R}$

For $m \geqslant 1$, let $\bar{e}_{m}$ be a copy of $\bar{e}=\left(e_{b}\right)_{1 \leqslant b \leqslant s}$. Set by definition $\bar{e}_{m}=\overline{0}$ if $m \leqslant 0$.

For $n \geqslant 1$, construct the objects $\mathcal{L}_{n}=\left(L_{n}, F_{n},[\pi]_{n}\right)$ of the category $\bmod { }^{h}(O)_{R}$ as follows. 
$L_{n}$ will be the free $R$-module of rank $2 n s$ with the basis consisting of all coordinates of the vectors $\bar{e}_{1}, \ldots, \bar{e}_{2 n}$. Define the linear maps $F_{n}: L_{n}^{(q)} \longrightarrow L_{n}$ and $V_{n}: L_{n} \longrightarrow L_{n}^{(q)}$ by the following relations, where $1 \leqslant m \leqslant n$ :

$$
\begin{aligned}
& F_{n}\left(\bar{e}_{2 m} \otimes 1\right)=\bar{e}_{2 m} D+\bar{e}_{2 m-1} \\
& V_{n}\left(\bar{e}_{2 m}\right)=\bar{e}_{2 m} \otimes C+\bar{e}_{2 m-1} \otimes 1 ; \\
& F_{n}\left(\bar{e}_{2 m-1} \otimes 1\right)=-\bar{e}_{2 m-1} C+\sum_{i \geqslant 0} \bar{e}_{2 m-2 i} Y_{i} ; \\
& V_{n}\left(\bar{e}_{2 m-1}\right)=-\bar{e}_{2 m-1} \otimes D+\sum_{i \geqslant 0} \bar{e}_{2 m-2 i} \otimes X_{i}
\end{aligned}
$$

where for $i \geqslant 0$, the matrices $X_{i}, Y_{i} \in M_{s}(R)$ are such that

- $C D+X_{0}=\pi^{h} I_{s}$ and $D C+Y_{0}=\pi^{h} I_{s}$;

- for $1 \leqslant i \leqslant h, X_{i}=Y_{i}=(-1)^{i}\left(\begin{array}{c}h \\ i\end{array}\right) \pi^{h-i} I_{s}$;

- for $i>h, X_{i}=Y_{i}=0$.

Lemma 3. For $i \geqslant 0, D X_{i}=Y_{i} D$.

Proof. It is obviously true if $i \geqslant 1$, because in this case $X_{i}=Y_{i}$ are just scalar matrices. If $i=0$ then $Y_{0} D=\left(\pi^{h} I_{s}-D C\right) D=D\left(\pi^{h} I_{s}-C D\right)=D X_{0}$. The lemma is proved.

Lemma 4. $\sum_{i \geqslant 0}[\pi]^{i}(\bar{e}) Y_{i}=0$.

Proof. We must prove that $\sum_{i \geqslant 0} \Pi^{i} Y_{i}=0$. But

$Y_{0}=-D C+\pi^{h} I_{s}=-\left(\pi I_{s}-\Pi\right)^{h}+\pi^{h} I_{s}=-\sum_{i \geqslant 1}(-1)^{i} \pi^{h-i}\left(\begin{array}{c}h \\ i\end{array}\right) \Pi^{i}=-\sum_{i \geqslant 1} \Pi^{i} Y_{i}$.

The lemma is proved.

For $1 \leqslant i \leqslant 2 n$, set $[\pi]_{n}\left(\bar{e}_{i}\right)=\bar{e}_{i-2}$.

Proposition 5. For any $n \geqslant 1, \mathcal{L}_{n}=\left(L_{n}, F_{n},[\pi]_{n}\right)$ is an object of the category $\bmod { }^{h}(O)_{R}$.

Proof. Clearly, $[\pi]_{n}$ is nilpotent. Therefore, $\pi \mathrm{id}_{L_{n}}-[\pi]_{n}$ is given by a nondegenerate matrix and it will be sufficient to verify the following two properties: 
a) $F_{n}[\pi]_{n}=[\pi]_{n}^{(q)} F_{n}$

b) $V_{n} F_{n}=\left(\pi \operatorname{id}_{L_{n}}-[\pi]_{n}\right)^{h}$.

Let $1 \leqslant m \leqslant n$.

Verify a):

$\left(F_{n}[\pi]_{n}\right)\left(\bar{e}_{2 m} \otimes 1\right)=[\pi]_{n}\left(F_{n}\left(\bar{e}_{2 m} \otimes 1\right)\right)=[\pi]_{n}\left(\bar{e}_{2 m} D+\bar{e}_{2 m-1}\right)=\bar{e}_{2 m-2} D+\bar{e}_{2 m-3}$ $\left([\pi]_{n}^{(q)} F_{n}\right)\left(\bar{e}_{2 m} \otimes 1\right)=F_{n}\left(\bar{e}_{2 m-2} \otimes 1\right)=\bar{e}_{2 m-2} D+\bar{e}_{2 m-3}$ $\left(F_{n}[\pi]_{n}\right)\left(\bar{e}_{2 m-1} \otimes 1\right)=[\pi]_{n}\left(-\bar{e}_{2 m-1} C+\sum_{i \geqslant 0} \bar{e}_{2 m-2 i} Y_{i}\right)=-\bar{e}_{2 m-3} C+\sum_{i \geqslant 0} \bar{e}_{2(m-1)-2 i} Y_{i}$ $\left([\pi]_{n}^{(q)} F_{n}\right)\left(\bar{e}_{2 m-1} \otimes 1\right)=F_{n}\left(\bar{e}_{2 m-3} \otimes 1\right)=-\bar{e}_{2 m-3} C+\sum_{i \geqslant 0} \bar{e}_{2(m-1)-2 i} Y_{i}$

Now verify b):

$$
\begin{gathered}
\left(V_{n} F_{n}\right)\left(\bar{e}_{2 m}\right)=F_{n}\left(V_{n}\left(\bar{e}_{2 m}\right)\right)=F_{n}\left(\bar{e}_{2 m} \otimes C\right)+F_{n}\left(\bar{e}_{2 m-1} \otimes 1\right) \\
=\bar{e}_{2 m} D C+\bar{e}_{2 m-1} C-\bar{e}_{2 m-1} C+\sum_{i \geqslant 0} \bar{e}_{2 m-2 i} Y_{i} \\
=\bar{e}_{2 m}\left(D C+Y_{0}\right)+\sum_{i \geqslant 1} \bar{e}_{2 m-2 i}(-1)^{i} \pi^{h-i}\left(\begin{array}{c}
h \\
i
\end{array}\right) \\
=\sum_{h \geqslant i \geqslant 0} \bar{e}_{2 m-2 i}(-1)^{i} \pi^{h-i}\left(\begin{array}{c}
h \\
i
\end{array}\right)=\left(\pi \mathrm{id}_{L_{n}}-[\pi]_{n}\right)^{h}\left(\bar{e}_{2 m}\right) ; \\
\left(V_{n} F_{n}\right)\left(\bar{e}_{2 m-1}\right)=F_{n}\left(V_{n}\left(\bar{e}_{2 m-1}\right)\right)=-F_{n}\left(\bar{e}_{2 m-1} \otimes D\right)+\sum_{i \geqslant 0} F_{n}\left(\bar{e}_{2 m-2 i} \otimes X_{i}\right) \\
=\bar{e}_{2 m-1} C D-\sum_{i \geqslant 0} \bar{e}_{2 m-2 i} Y_{i} D+\sum_{i \geqslant 0}\left(\bar{e}_{2 m-2 i} D X_{i}+\bar{e}_{2 m-2 i-1} X_{i}\right) \\
=\bar{e}_{2 m-1}\left(C D+X_{0}\right)+\sum_{i \geqslant 1} \bar{e}_{2 m-2 i-1} X_{i}+\sum_{i \geqslant 0} \bar{e}_{2 m-2 i}\left(-Y_{i} D+D X_{i}\right) \\
=\sum_{i \geqslant 0} \bar{e}_{2 m-1-2 i}(-1)^{i} \pi^{h-i}\left(\begin{array}{c}
h \\
i
\end{array}\right)=\left(\pi \mathrm{id}_{L_{n}}-[\pi]_{n}\right)^{h}\left(\bar{e}_{2 m-1}\right) .
\end{gathered}
$$

The proposition is proved. 
Notice that for any $n \geqslant 1$, we have natural strict embeddings $i_{n}: \mathcal{L}_{n} \longrightarrow \mathcal{L}_{n+1}$ in the category $\bmod ^{h}(O)_{R}$. Then the above proposition implies the following corollary.

Corollary 6. The inductive system $\left\{\mathcal{L}_{n}, i_{n}\right\}_{n \geqslant 1}$ is a $[\pi]$-divisible group in the category $\bmod ^{h}(O)_{R}$.

\subsection{Epimorphic map $f: \mathcal{L}_{N} \longrightarrow \mathcal{L}$}

For $1 \leqslant m \leqslant N$, set $f\left(\bar{e}_{2 m}\right)=[\pi]^{N-m}(\bar{e})=\bar{e} \Pi^{N-m}$ and $f\left(\bar{e}_{2 m-1}\right)=\overline{0}$. This gives the $R$-linear map $f: L_{N} \longrightarrow L$. This map is epimorphic because $f\left(\bar{e}_{2 N}\right)=$ $\bar{e}$. It remains to verify that $f$ is a morphism in the category $\bmod ^{h}(O)_{R}$.

Proposition 7. a) $f[\pi]=[\pi]_{N} f$;

b) $f^{(q)} F=F_{N} f$.

Proof. Let $1 \leqslant m \leqslant N$. Verify a):

$$
\begin{aligned}
& (f[\pi])\left(\bar{e}_{2 m}\right)=[\pi]^{N+1-m}(\bar{e})=f\left(\bar{e}_{2 m-2}\right)=\left([\pi]_{N} f\right)\left(\bar{e}_{2 m}\right) \\
& (f[\pi])\left(\bar{e}_{2 m-1}\right)=\overline{0}=f\left(\bar{e}_{2 m-3}\right)=\left([\pi]_{N} f\right)\left(\bar{e}_{2 m-1}\right) .
\end{aligned}
$$

Now verify b):

$$
\begin{aligned}
& \left(F_{N} f\right)\left(\bar{e}_{2 m} \otimes 1\right)=f\left(\bar{e}_{2 m} D\right)+f\left(\bar{e}_{2 m-1}\right)=[\pi]^{N-m}(\bar{e}) D=\bar{e} \Pi^{N-m} D \\
& \left(f^{(q)} F\right)\left(\bar{e}_{2 m} \otimes 1\right)=F\left([\pi]^{N-m} \bar{e} \otimes 1\right)=F\left(\bar{e} \otimes \Pi^{(q)^{N-m}}\right)=\bar{e} D \Pi^{(q)^{N-m}}
\end{aligned}
$$

and use that $\Pi D=D \Pi^{(q)}$.

Finally, $\left(f^{(q)} F\right)\left(\bar{e}_{2 m-1} \otimes 1\right)=0$ and

$$
\left(F_{N} f\right)\left(\bar{e}_{2 m-1} \otimes 1\right)=f\left(-\bar{e}_{2 m-1} C+\sum_{i \geqslant 0} \bar{e}_{2 m-2 i} Y_{i}\right)=[\pi]^{N-m}\left(\sum_{i \geqslant 0}[\pi]^{i}(\bar{e}) Y_{i}\right)=0
$$

by Lemma 4 . The proposition is proved.

\section{Ramification estimates.}

Suppose $h \in \mathbb{N}$ and $G \in \mathrm{MG}^{h}(O)_{K}$, where $O=\mathbb{F}_{q}[[\pi]]$ is the valuation ring of the field of formal Laurent series $K_{0}=\mathbb{F}_{q}((\pi)), K=k((u))$ is an algebraic extension of $K_{0}$ with perfect residue field $k, R=O_{K}$ is the valuation ring of $K$ 
and $e$ is the ramification index of the extension $K / K_{0}$. We denote by $v_{K}$ the valuation on $K$ such that $v_{K}\left(K^{*}\right)=\mathbb{Z}$.

Theorem B. If $N \in \mathbb{N}$ is such that $\pi^{N} G=0$ then for

$$
v>e\left(N-1+\frac{h q}{q-1}\right)-1
$$

the ramification subgroup $\Gamma_{K}^{(v)}$ acts trivially on $G$.

The proof of Theorem B follows the strategy from [Ab6] (where the case $h=1$ was considered) and will be given in nn.3.1-3.4 below.

\subsection{Auxiliary field $K_{\alpha},[\mathrm{Ab} 4, \mathrm{n} .1 .5]$}

Let $\alpha$ be a rational positive number with zero $p$-adic valuation. Then there are $m \in \mathbb{N}, \operatorname{gcd}(m, p)=1$, and $M \in \mathbb{N}$ such that $\alpha=m /\left(q^{M}-1\right)$. Notice that for a given $\alpha$, the corresponding numbers $m$ and $M$ are not unique and can be chosen to be arbitrarily large if necessary. Then there is a field extension $K_{\alpha}$ of $K$ with the same residue field $k$ such that $\left[K_{\alpha}: K\right]=q^{M}$ and its Herbrand function equals

$$
\varphi_{K_{\alpha} / K}(x)= \begin{cases}x, & \text { if } 0 \leqslant x \leqslant \alpha ; \\ \alpha+\frac{x-\alpha}{q^{M}}, & \text { if } x \geqslant \alpha .\end{cases}
$$

From the construction of this field extension $K_{\alpha} / K$, cf.[Ab4, n.1.5], it follows the existence of a uniformising element $u_{\alpha}$ of $K_{\alpha}$ such that $v_{K}\left(u-u_{\alpha}^{q^{M}}\right)=$ $1+\alpha\left(1-q^{-M}\right)$.

Introduce the field isomorphism $\kappa_{\alpha}: K \longrightarrow K_{\alpha}=k\left(\left(u_{\alpha}\right)\right)$ such that $\kappa_{\alpha}(u)=$ $u_{\alpha}$ and $\left.\kappa_{\alpha}\right|_{k}=\mathrm{id}$. We have the following properties:

- $\forall a \in R, v_{K}\left(a-\kappa_{\alpha}(a)^{q^{M}}\right) \geqslant 1+\alpha\left(1-q^{-M}\right)$;

- $\forall a \in K, v_{K}\left(a-\kappa_{\alpha}(a)^{q^{M}}\right) \geqslant v_{K}(a)+\alpha\left(1-q^{-M}\right)$.

Here and everywhere below in the paper we shall use the following notation:

— for $a \in K, \tilde{a}:=a-\kappa_{\alpha}(a)^{q^{M}}$

$-\pi_{\alpha}:=\kappa_{\alpha}(\pi)$

$-\alpha^{*}=\alpha\left(1-q^{-M}\right)$. 
The role of the fields $K_{\alpha}$ in obtaining ramification estimates can be explained as follows.

First, remind the definition of the biggest ramification number $v\left(E_{2} / E_{1}\right)$, where $E_{1} \subset E_{2}$ are finite extensions of $K$ in $K_{\text {sep }}$. By definition, $v\left(E_{2} / E_{1}\right)$ is the second coordinate of the last corner point of the Herbrand function $\varphi_{E_{2} / E_{1}}$. Equivalently, the ramification subgroups $\Gamma_{E_{1}}^{(v)}$ of $\Gamma_{E_{1}}=\operatorname{Gal}\left(K_{\mathrm{sep}} / E_{1}\right)$ act trivially on $E_{2}$ if and only if $v>v\left(E_{2} / E_{1}\right)$. For example, $v\left(K_{\alpha} / K\right)=\alpha$.

Now consider a field isomorphism $\bar{\kappa}_{\alpha}: K_{\text {sep }} \longrightarrow K_{\text {sep }}$ such that $\left.\bar{\kappa}_{\alpha}\right|_{K}=\kappa_{\alpha}$.

Proposition 8. Let $E$ be a finite field extension of $K$ in $K_{\mathrm{sep}}$ and let $E_{\alpha}=$ $\bar{\kappa}_{\alpha}(E)$. Then:

a) if $E \subset E_{\alpha}$ then $v(E / K) \leqslant \alpha$;

b) if there is a field extension $K^{\prime} \supset K$ such that $E E_{\alpha}=K^{\prime} E_{\alpha}$ and $v\left(K^{\prime} / K\right)>\alpha$ then $v(E / K)=v\left(K^{\prime} / K\right)$.

Proof. a) Suppose $v(E / K)=v_{0}>\alpha$. Then $\Gamma_{K}^{\left(v_{0}\right)}$ acts non-trivially on $E$, acts trivially on $K_{\alpha}$ (because $v\left(K_{\alpha} / K\right)=\alpha$ ) and, therefore, acts non-trivially on $E K_{\alpha} \subset E_{\alpha}$. On the other hand, $\Gamma_{K}^{\left(v_{0}\right)}=\Gamma_{K_{\alpha}}^{\left(v_{1}\right)}$ with $v_{0}=\varphi_{K_{\alpha} / K}\left(v_{1}\right)$ by the definition of the Herbrand function, cf. [Se]. But then $v_{1}>v_{0}$ (use the explicit form of $\left.\varphi_{K_{\alpha} / K}\right)$ and $\Gamma_{K_{\alpha}}^{\left(v_{1}\right)}$ must act trivially on $E_{\alpha}$ because $v\left(E_{\alpha} / K_{\alpha}\right)=$ $v(E / K)=v_{0}<v_{1}$. The contradiction. So, $v(E / K) \leqslant \alpha$ and a) is proved.

b) Apply the composition property of Herbrand's functions

$$
\varphi_{E_{\alpha} / K}(x)=\varphi_{K_{\alpha} / K}\left(\varphi_{E_{\alpha} / K_{\alpha}}(x)\right)
$$

where $x \geqslant 0$, to their last corner points to obtain

$$
v\left(E_{\alpha} / K\right)=\max \left\{\alpha, \varphi_{K_{\alpha} / K}\left(v\left(E_{\alpha} / K_{\alpha}\right)\right)\right\} .
$$

This gives that $v(E / K)>\alpha$. Indeed, otherwise,

$$
\varphi_{K_{\alpha} / K}(v(E / K))=\varphi_{K_{\alpha} / K}\left(v\left(E_{\alpha} / K_{\alpha}\right)\right) \leqslant \alpha,
$$

implies that $v\left(E_{\alpha} / K\right)=\alpha$ and, therefore,

$$
v\left(E E_{\alpha} / K\right)=\max \left\{v(E / K), v\left(E_{\alpha} / K\right)\right\}=\alpha<v\left(K^{\prime} E_{\alpha} / K\right) .
$$


The contradiction. Therefore, $\alpha<v\left(E_{\alpha} / K\right)<v(E / K)$ (use the above explicit formula for $\left.\varphi_{K_{\alpha} / K}\right)$ and $v(E / K)=v\left(E E_{\alpha} / K\right)=v\left(K^{\prime} E_{\alpha} / K\right)=v\left(K^{\prime} / K\right)$. The part $b$ ) is proved.

3.2. By Theorem A we can assume that $G$ is the $\pi^{N}$-torsion part of a $\pi$ divisible group in the category $\mathrm{MG}^{h}(O)_{K}$. So, if $G$ comes from $\mathcal{L}=(L, F,[\pi]) \in$ mod ${ }^{h}(O)_{R}$ then we can choose an $R$-basis of $L$ consisting of elements of vectors $\bar{e}_{1}, \ldots, \bar{e}_{N}$, where for $i=1, \ldots, N$, each $\bar{e}_{i}$ is a copy of $\bar{e}=\left(e_{1}, \ldots, e_{s}\right)$ and it holds $[\pi]\left(\bar{e}_{1}\right)=\overline{0},[\pi]\left(\bar{e}_{2}\right)=\bar{e}_{1}, \ldots,[\pi]\left(\bar{e}_{N}\right)=\bar{e}_{N-1}$. Then the structure of an object of the category $\bmod ^{h}(O)_{R}$ on $\mathcal{L}$ is given via matrices $C_{1}, \ldots, C_{N} \in M_{s}(R)$, where $\operatorname{det}\left(C_{1}\right) \neq 0$ and

$$
\begin{aligned}
& F\left(\bar{e}_{1} \otimes 1\right) C_{1}=\pi^{h} \bar{e}_{1} \\
& F\left(\bar{e}_{2} \otimes 1\right) C_{1}+F\left(\bar{e}_{1} \otimes 1\right) C_{2}=\pi^{h} \bar{e}_{2}-\left(\begin{array}{c}
h \\
1
\end{array}\right) \pi^{h-1} \bar{e}_{1} \\
& \cdots \cdots \cdots \cdots \cdots \cdots \cdots \cdots \\
& \quad \begin{array}{c}
F\left(\bar{e}_{N} \otimes 1\right) C_{1}+\cdots+F\left(\bar{e}_{1} \otimes 1\right) C_{N}=\pi^{h} \bar{e}_{N} \\
+\cdots+(-1)^{i}\left(\begin{array}{c}
h \\
i
\end{array}\right) \pi^{h-i} \bar{e}_{N-i}+\cdots+(-1)^{h} \bar{e}_{N-h}
\end{array}
\end{aligned}
$$

with the agreement $\bar{e}_{i}=\overline{0}$ if $i \leqslant 0$.

Then $G$ appears as the set of $K_{\text {sep}}$-points of the $K$-scheme $\mathcal{B}$ given by the equations

$$
\begin{aligned}
& \bar{X}_{1}^{(q)} C_{1}=\pi^{h} \bar{X}_{1} \\
& \bar{X}_{2}^{(q)} C_{1}+\bar{X}_{1}^{(q)} C_{2}=\pi^{h} \bar{X}_{2}-\left(\begin{array}{c}
h \\
1
\end{array}\right) \pi^{h-1} \bar{X}_{1}
\end{aligned}
$$

$$
\begin{aligned}
& \bar{X}_{N}^{(q)} C_{1}+\cdots+\bar{X}_{1}^{(q)} C_{N}=\pi^{h} \bar{X}_{N} \\
&+\cdots+(-1)^{i}\left(\begin{array}{c}
h \\
i
\end{array}\right) \pi^{h-i} \bar{X}_{N-i}+\cdots+(-1)^{h} \bar{X}_{N-h}
\end{aligned}
$$

Here $\bar{X}_{1}, \ldots, \bar{X}_{N}$ are copies of the vector $\bar{X}$, which contains as its coordinates $s$ independent variables, and by definition $\bar{X}_{i}=\overline{0}$ if $i \leqslant 0$. As earlier, each $\bar{X}_{i}^{(q)}$ is obtained from $\bar{X}_{i}$ by raising all its entries to $q$-th power. 
3.3. Consider the $K_{\alpha}$-scheme $\mathcal{B}_{\alpha}$ given as Spec $K_{\alpha}\left[\bar{Y}_{1}, \ldots, \bar{Y}_{N}\right]$ with equations

$$
\begin{aligned}
& \bar{Y}_{1}^{(q)} \kappa_{\alpha}\left(C_{1}\right)=\pi_{\alpha}^{h} \bar{Y}_{1} \\
& \bar{Y}_{2}^{(q)} \kappa_{\alpha}\left(C_{1}\right)+\bar{Y}_{1}^{(q)} \kappa_{\alpha}\left(C_{2}\right)=\pi_{\alpha}^{h} \bar{Y}_{2}-\left(\begin{array}{c}
h \\
1
\end{array}\right) \pi_{\alpha}^{h-1} \bar{Y}_{1}
\end{aligned}
$$

$(2)$

$$
\begin{aligned}
\bar{Y}_{N}^{(q)} \kappa_{\alpha}\left(C_{1}\right)+\cdots+ & \bar{Y}_{1}^{(q)} \kappa_{\alpha}\left(C_{N}\right)=\pi_{\alpha}^{h} \bar{Y}_{N}+\cdots+(-1)^{i}\left(\begin{array}{c}
h \\
i
\end{array}\right) \pi_{\alpha}^{h-i} \bar{Y}_{N-i} \\
& +\cdots+(-1)^{h} \bar{Y}_{N-h}
\end{aligned}
$$

Here $\bar{Y}_{1}, \ldots, \bar{Y}_{N}$ are copies of the vector $\bar{Y}$, which contains as its coordinates $s$ independent variables, and by definition $\bar{Y}_{i}=\overline{0}$ if $i \leqslant 0$.

As earlier in n.3.1, consider the field isomorphism $\bar{\kappa}_{\alpha}: K_{\text {sep }} \longrightarrow K_{\text {sep }}$, which extends the field isomorphism $\kappa_{\alpha}$. Clearly, $\bar{\kappa}_{\alpha}$ induces a one-to-one map between the points $G=\mathcal{B}\left(K_{\mathrm{sep}}\right)$ and $G_{\alpha}:=\mathcal{B}_{\alpha}\left(K_{\mathrm{sep}}\right)$. In particular, if $E=K(G)$ (resp. $E_{\alpha}=K_{\alpha}\left(G_{\alpha}\right)$ ) is the field of definition of all points of $G$ (resp. $G_{\alpha}$ ) over $K$ (resp. $\left.K_{\alpha}\right)$, then $\bar{\kappa}_{\alpha}(E)=E_{\alpha}$.

Lemma 9. a) If $\alpha^{*}>e\left(N-1+\frac{h q}{q-1}\right)-1$ and $\left(\bar{Y}_{1}^{0}, \ldots, \bar{Y}_{N}^{0}\right) \in G_{\alpha}$ then there is a unique $\left(\bar{X}_{1}^{0}, \ldots, \bar{X}_{N}^{0}\right) \in G$ such that $v_{K}\left(\bar{X}_{i}^{0}-\bar{Y}_{i}^{0 q^{M}}\right)>\frac{e h}{q-1}$ for all $1 \leqslant i \leqslant N$.

b) With the above notation the correspondence

$$
\left(\bar{Y}_{1}^{0}, \ldots, \bar{Y}_{N}^{0}\right) \mapsto\left(\bar{X}_{1}^{0}, \ldots, \bar{X}_{N}^{0}\right)
$$

gives a one-to-one map between the points of $G_{\alpha}$ and $G$.

Lemma 9 implies the following corollary.

Corollary 10. If $\alpha^{*}>e\left(N-1+\frac{h q}{q-1}\right)-1$ then $E K_{\alpha}=E_{\alpha}$.

Proof of corollary. Denote by $\eta: G_{\alpha} \longrightarrow G$ the one-to-one map from Lemma 9. Clearly, for any $\tau \in \Gamma_{K_{\alpha}}=\operatorname{Gal}\left(K_{\mathrm{sep}} / K_{\alpha}\right)$ and $\bar{Y}^{0} \in G_{\alpha}$, it holds $\tau\left(\eta\left(\bar{Y}^{0}\right)\right)=$ $\eta\left(\tau\left(\bar{Y}^{0}\right)\right)$. Therefore, $\operatorname{Gal}\left(K_{\mathrm{sep}} / E_{\alpha}\right)=\left\{\tau \in \Gamma_{K_{\alpha}} \mid \tau \bar{Y}^{0}=\bar{Y}^{0}, \forall \bar{Y}^{0} \in G_{\alpha}\right\}$ $=\left\{\tau \in \Gamma_{K_{\alpha}} \mid \tau \bar{X}^{0}=\bar{X}^{0}, \forall \bar{X}^{0} \in G\right\}=\operatorname{Gal}\left(K_{\mathrm{sep}} / E K_{\alpha}\right)$. The corollary is proved. Proof of Lemma 9 First, prove the part a.)

For $1 \leqslant i \leqslant N$, let $\bar{Z}_{i}=\bar{X}_{i}-\bar{Y}_{i}^{0\left(q^{M}\right)}, \widetilde{C}_{i}=C_{i}-\kappa_{\alpha}\left(C_{i}\right)^{\left(q^{M}\right)}$ and (as earlier) $\widetilde{\pi^{i}}=\pi^{i}-\kappa_{\alpha}\left(\pi^{i}\right)^{q^{M}}$. Then $\bar{Z}_{1}, \ldots, \bar{Z}_{N}$ satisfy the following equations (where by 
definition $\bar{Z}_{i}=\overline{0}$ if $\left.i \leqslant 0\right)$

$$
\begin{aligned}
& \bar{Z}_{1}^{(q)} C_{1}=\pi^{h} \bar{Z}_{1}+\bar{F}_{1} \\
& \bar{Z}_{2}^{(q)} C_{1}+\bar{Z}_{1}^{(q)} C_{2}=\pi^{h} \bar{Z}_{2}-\left(\begin{array}{c}
h \\
1
\end{array}\right) \pi^{h-1} \bar{Z}_{1}+\bar{F}_{2}
\end{aligned}
$$

$$
\begin{gathered}
\bar{Z}_{N}^{(q)} C_{1}+\cdots+\bar{Z}_{1}^{(q)} C_{N}=\pi^{h} \bar{Z}_{N}+\cdots+(-1)^{i}\left(\begin{array}{c}
h \\
i
\end{array}\right) \pi^{h-i} \bar{Z}_{N-i} \\
+\cdots+(-1)^{h} \bar{Z}_{N-h}+\bar{F}_{N}
\end{gathered}
$$

where

$$
\begin{aligned}
& \bar{F}_{1}=\widetilde{\pi^{h}} \bar{Y}_{1}^{0\left(q^{M}\right)}-\bar{Y}_{1}^{0\left(q^{M+1}\right)} \widetilde{C}_{1} \\
& \bar{F}_{2}=\widetilde{\pi^{h}} \bar{Y}_{2}^{0\left(q^{M}\right)}-\left(\begin{array}{c}
h \\
1
\end{array}\right) \widetilde{\pi^{h-1}} \bar{Y}_{1}^{0\left(q^{M}\right)}-\left(\bar{Y}_{2}^{0\left(q^{M+1}\right)} \widetilde{C}_{1}+\bar{Y}_{1}^{0\left(q^{M}\right)} \widetilde{C}_{2}\right) \\
& \cdots \ldots \ldots \ldots \ldots \ldots \ldots . . . \\
& \bar{F}_{N}=\widetilde{\pi^{h}} \bar{Y}_{N}^{0\left(q^{M}\right)}+\cdots+(-1)^{i}\left(\begin{array}{c}
h \\
i
\end{array}\right) \widetilde{\pi^{h-i}} \bar{Y}_{N-i}^{0\left(q^{M}\right)}+\cdots+(-1)^{h-1} h \tilde{\pi} \bar{Y}_{N-h+1}^{0\left(q^{M}\right)}- \\
& -\left(\bar{Y}_{N}^{0\left(q^{M+1}\right)} \widetilde{C}_{1}+\cdots+\bar{Y}_{1}^{0\left(q^{M+1}\right)} \widetilde{C}_{N}\right)
\end{aligned}
$$

Clearly, the above formulas imply that

$$
v_{K}\left(\bar{F}_{i}\right)=\min \left\{v_{K}\left(\text { coordinates of } \bar{F}_{i}\right)\right\} \geqslant \alpha^{*}+1>e\left(N-1+\frac{h q}{q-1}\right) .
$$

Then the statement a) of Lemma 9 is equivalent to the existence of a unique solution $\bar{Z}_{1}^{0}, \ldots, \bar{Z}_{N}^{0} \in K_{\text {sep }}^{s}$ of system (3) such that for all $1 \leqslant i \leqslant N, v_{K}\left(\bar{Z}_{i}^{0}\right)>$ $\frac{e h}{q-1}$.

This follows easily by induction from the following lemma.

Lemma 11. Suppose $\bar{Z}$ is a vector with s independent variables as its coordinates and $\bar{a} \in K_{\text {sep }}^{s}$ is such that $v_{K}(\bar{a})>\frac{e h q}{q-1}$. Then the system of equations

$$
\bar{Z}^{(q)} C_{1}-\pi^{h} \bar{Z}=\bar{a}
$$

has a unique solution $\bar{Z}^{0} \in K_{\mathrm{sep}}^{s}$ such that $v_{K}\left(\bar{Z}^{0}\right)=v_{K}(\bar{a})-e$; in addition, all coordinates of $\bar{Z}^{0}$ belong to the field of definition of $\bar{a}$ over $K$.

Proof of Lemma 11. The existence is given by the following explicit formula $\bar{Z}^{0}=-\pi^{-h} \bar{a}-\pi^{-h(1+q)} \bar{a}^{(q)} C_{1}-\ldots-\pi^{-h\left(1+\ldots+q^{n+1}\right)} \bar{a}^{\left(q^{n+1}\right)} C_{1}^{\left(q^{n}\right)} C_{1}^{\left(q^{n-1}\right)} \ldots C_{1}-\ldots$ 
Suppose $\bar{Z}^{1} \neq \bar{Z}^{0}$ is another solution with $v_{K}\left(\bar{Z}^{1}\right)>\frac{e h}{q-1}$. Then for $\bar{W}=$ $\bar{Z}^{0}-\bar{Z}^{1}$ we have $v_{K}(\bar{W})>\frac{e h}{q-1}$ and $\bar{W}^{(q)} C_{1}=\pi^{h} \bar{W}$. But then $v_{K}\left(\pi^{h} \bar{W}\right)=$ $v_{K}\left(\bar{W}^{(q)} C_{1}\right) \geqslant v_{K}\left(\bar{W}^{(q)}\right)=v_{K}(\bar{W})+(q-1) v_{K}(\bar{W})>v_{K}(\bar{W})+e h=v_{K}\left(\pi^{h} \bar{W}\right)$. The contradiction. Lemma 11 is proved.

Continue the proof of lemma 9. Its part b) follows from the following observation. Any two solutions $\left(\bar{X}_{1}^{0}, \ldots, \bar{X}_{N}^{0}\right)$ and $\left(\bar{X}_{1}^{1}, \ldots, \bar{X}_{N}^{1}\right)$ of (1) such that for $1 \leqslant i \leqslant N, v_{K}\left(\bar{X}_{i}^{0}-\bar{X}_{i}^{1}\right)>e h /(q-1)$, must coincide. This can be proved again by induction from the uniqueness property of Lemma 11. Lemma 9 is proved.

3.4. Finally, suppose that

$$
v=v(E / K)>e\left(N-1+\frac{q h}{q-1}\right)-1 .
$$

Choose $\alpha \in \mathbb{Q}, \alpha>0$ with zero $p$-adic valuation and a corresponding big enough $M \in \mathbb{N}$, cf. n.3.1, such that

$$
v>\alpha>\alpha^{*}>e\left(N-1+\frac{q h}{q-1}\right)-1 .
$$

Then by Corollary 10 we have $E_{\alpha}=E K_{\alpha}$ and, therefore, Proposition 8 implies that $v \leqslant \alpha$. The contradiction. Therefore, the above assumption about $v=$ $v(E / K)$ is false and Theorem B is completely proved.

\section{Computation of an upper ramification number}

As earlier, $h, N \in \mathbb{N}, K=k((u))$ with perfect $k$ of characteristic $p, q$ is a power of $p, K_{0}=\mathbb{F}_{q}((\pi))$ is a closed subfield in $K, R$ and $O$ are valuation rings in $K$ and $K_{0}$, respectively, and $e=e\left(K / K_{0}\right)$ is the ramification index of $K / K_{0}$.

\subsection{The statement of Theorem $C$}

Introduce $\mathcal{L}=(L, F,[\pi]) \in \bmod { }^{h}(O)_{R}$ as follows.

Let $L$ be a free $R$-module with the basis $e_{1}, \ldots, e_{N}, e_{1}^{0}, \ldots, e_{N}^{0}$. Define $[\pi] \in$ $\operatorname{End}_{R} L$ by the relations $[\pi]\left(e_{n}^{0}\right)=e_{n-1}^{0}$ and $[\pi]\left(e_{n}\right)=e_{n-1}$, where $1<n \leqslant N$. Define an $R$-linear morphism $F: L^{(q)} \longrightarrow L$ by the following relations:

$$
F\left(e_{n}^{0} \otimes 1\right)=e_{n}^{0}, \quad F\left(e_{n} \otimes 1\right)=\sum_{0 \leqslant j \leqslant h}(-1)^{j}\left(\begin{array}{l}
h \\
j
\end{array}\right) \pi^{h-j} e_{n-j}-u e_{n}^{0},
$$


where $1 \leqslant n \leqslant N$, and by definition $e_{n}=e_{n}^{0}=0$ if $n \leqslant 0$. Clearly, for any $1 \leqslant n \leqslant N,\left(\pi \operatorname{id}_{L}-[\pi]\right)^{h}\left(e_{n}^{0}\right)$ is an $R$-linear combination of $F\left(e_{i}^{0} \otimes 1\right)=e_{i}^{0}$, $1 \leqslant i \leqslant n$, and also $\left(\pi \operatorname{id}_{L}-[\pi]\right)^{h}\left(e_{n}\right)=F\left(e_{n} \otimes 1\right)+u e_{n}^{0} \in \operatorname{Im} F$. Therefore, $\mathcal{L}=(L, F,[\pi]) \in \bmod ^{h}(O)_{R}$.

Let $\mathcal{L}^{(0)}:=\left(L^{(0)}, F^{(0)},[\pi]^{(0)}\right)$, where $L^{(0)}$ is the submodule of $L$ generated by $e_{1}^{0}, \ldots, e_{N}^{0}$ and $F^{(0)}$ and $[\pi]^{(0)}$ are induced by $F$ and $[\pi]$, respectively. Then $\mathcal{L}^{(0)} \in$ $\bmod { }^{0}(O)_{R} \subset \bmod ^{h}(O)_{R}$ and we have a natural embedding of $\mathcal{L}^{(0)}$ into $\mathcal{L}$ in the category $\bmod ^{h}(O)_{R}$. This embedding is strict and gives rise to the following short exact sequence in $\bmod ^{h}(O)_{R}, 0 \longrightarrow \mathcal{L}^{(0)} \longrightarrow \mathcal{L} \longrightarrow \mathcal{L}^{(h)} \longrightarrow 0$, where $\mathcal{L}^{(h)}=\left(L^{(h)}, F^{(h)},[\pi]^{(h)}\right) \in \bmod ^{h}(O)_{R}, L^{(h)}$ is the free $R$-module with basis $e_{1}^{1}, \ldots, e_{N}^{1}, \quad[\pi]^{(h)}\left(e_{n}^{1}\right)=e_{n-1}^{1}$ and $F^{(h)}\left(e_{n}^{1}\right)=\left(\pi \operatorname{id}_{L^{(h)}}-[\pi]^{(h)}\right)^{h} e_{n}^{1}$ with $1 \leqslant n \leqslant N$ and $e_{0}^{1}=0$.

Consider the corresponding short exact sequence of $\Gamma_{K}$-modules

$$
0 \longrightarrow \mathcal{M}_{\Gamma}\left(\mathcal{L}^{(h)}\right) \longrightarrow \mathcal{M}_{\Gamma}(\mathcal{L}) \longrightarrow \mathcal{M}_{\Gamma}\left(\mathcal{L}^{(0)}\right) \longrightarrow 0
$$

Clearly $G^{(0)}=\mathcal{M}_{\Gamma}\left(\mathcal{L}^{(0)}\right)$ is just the cyclic module $O / \pi^{N}$ with the trivial $\Gamma_{K^{-}}$ action.

Remark. Though we do not need it, notice that for $h=1$, the Galois module structure on $G^{(1)}=\mathcal{M}_{\Gamma}\left(\mathcal{L}^{(1)}\right)$ is induced by the restriction to $\Gamma_{K}$ of the LubinTate character $\chi_{L T}: \Gamma_{K_{0}} \longrightarrow O^{*}$. In equivalent terminology, $G^{(1)}$ is the $\pi^{N_{-}}$ torsion of the Carlitz module over $O$. Then $\mathcal{L}^{(h)}$ appears as $h$-th tensor power of $\mathcal{L}^{(1)}$ and, therefore, the action of $\Gamma_{K}$ on the corresponding $G^{(h)}=\mathcal{M}_{\Gamma}\left(\mathcal{L}^{(h)}\right)$ is induced by the restriction to $\Gamma_{K}$ of the character $\chi_{L T}^{h}$.

Theorem C. Suppose $G=\mathcal{M}_{\Gamma}(\mathcal{L})$ and $\left(\begin{array}{c}-h \\ N-1\end{array}\right) \not \equiv 0 \bmod p$. Then the ramification subgroups $\Gamma_{K}^{(v)}$ act trivially on $G$ if and only if $v>e(N-1+h q /(q-1))-1$.

Remark. If $h=1$ then the above result has an analogue in the mixed characteristic case. Namely, if $K \supset \mathbb{Q}_{p}$ then there is a finite flat group scheme $\mathcal{G}$ over $R=O_{K}$ such that $\mathcal{G}=\operatorname{Spec} A, A=\oplus_{0 \leqslant i<p^{N}} R\left[\sqrt[p^{N}]{v^{i}}\right]$ and $v=1+u$, where $u$ is a uniformising element of $K$. This group scheme appears as an extension of the constant etale group scheme $\left(\mathbb{Z} / p^{N} \mathbb{Z}\right)_{R}$ over $R$ via the constant multiplicative group scheme $\mu_{p^{N}}=\operatorname{Spec} R[X] /\left(X^{p^{N}}-1\right)$ over $R$. One can verify that $\Gamma_{K}^{(v)}$ acts trivially on $\mathcal{G}\left(K_{\mathrm{sep}}\right)$ if and only if $v>e(N+1 /(p-1))-1$, i.e. Fontaine's estimate from [Fo1] is sharp. In the equicharacteristic case the above Theorem 
C shows that under the additional condition $\left(\begin{array}{c}-h \\ N-1\end{array}\right) \not \equiv 0 \bmod p$ the estimate from Theorem B is sharp. If this additional condition does not hold one should expect the existence of better ramification estimates.

Proof of Theorem $C$. The proof is given below in the remaining part of the paper.

4.2. Let $X_{1}, \ldots, X_{N}, Y_{1}, \ldots, Y_{N}$ be independent variables. Then the $O\left[\Gamma_{K}\right]$ module $G$ appears as the set of all solutions $g=\left(Y_{1}^{0}, \ldots, Y_{N}^{0}, X_{1}^{0}, \ldots, X_{N}^{0}\right) \in K_{\text {sep }}^{2 N}$ of the following system of equations:

$$
X_{n}^{q}=X_{n}, \quad Y_{n}^{q}=\sum_{0 \leqslant j \leqslant h}(-1)^{j}\left(\begin{array}{l}
h \\
j
\end{array}\right) \pi^{h-j} Y_{n-j}-u X_{n}
$$

where $1 \leqslant n \leqslant N$ and by definition $X_{n}=Y_{n}=0$ if $n \leqslant 0$. Notice that the structure of $O$-module on this set of solutions is induced by the usual addition and the action of $O$ is given by the relations $[\pi]\left(X_{n}\right)=X_{n-1},[\pi]\left(Y_{n}\right)=Y_{n-1}$ and for any $\alpha \in \mathbb{F}_{q},[\alpha]\left(X_{n}\right)=\alpha X_{n},[\alpha]\left(Y_{n}\right)=\alpha Y_{n}$. Let $g \in G$ be such that $X_{1}(g)=\cdots=X_{N}(g)=1$. Then its field of definition $K(g)$ over $K$ appears in the form $K\left(Y_{1}^{0}, \ldots, Y_{N}^{0}\right)$, where $\left(Y_{1}^{0}, \ldots, Y_{N}^{0}\right) \in K_{\text {sep }}^{N}$ is a solution of the following system of equations

$$
Y_{n}^{q}-\pi^{h} Y_{n}=\sum_{1 \leqslant j \leqslant h}(-1)^{j-1}\left(\begin{array}{c}
h \\
j
\end{array}\right) \pi^{h-j} Y_{n-j}-u, \quad 1 \leqslant n \leqslant N,
$$

Notice that for above choice of $g \in G$, the field of definition $K(G)$ of all points of $G$ over $K$ is equal to the composite $K(g) K\left(G^{(h)}\right)$, where $K\left(G^{(h)}\right)$ is the field of definition of all points of $G^{(h)}=\mathcal{M}_{\Gamma}\left(\mathcal{L}^{(h)}\right)$ over $K$. (Use that any point of $G$ appears in the form $[o] g+g_{1}$, where $o \in O$ and $g_{1} \in G^{(h)}$.)

\subsection{A property of $K\left(G^{(h)}\right)$}

Use the notation and definitions from n.3.1.

Proposition 12. There is $\delta(N, h)>0$ such that if $\alpha^{*}>e\left(N-1+\frac{h}{q-1}\right)-$ $\delta(N, h)$ then $K\left(G^{(h)}\right) K_{\alpha}=\bar{\kappa}_{\alpha}\left(K\left(G^{(h)}\right)\right)$.

Proof. The elements of $G^{(h)}$ are solutions $\left(X_{1}^{0}, \ldots, X_{N}^{0}\right) \in K_{\text {sep }}^{N}$ of the following system of equations (where, as usually, $X_{i}=0$ if $i<0$ ): 


$$
\begin{aligned}
& X_{1}^{q}=\pi^{h} X_{1} \\
& X_{2}^{q}=\pi^{h} X_{2}-\left(\begin{array}{c}
h \\
1
\end{array}\right) \pi^{h-1} X_{1} \\
& \cdots \cdots \cdots \cdots \cdots \cdots \cdots \cdots \cdots \cdots \cdots \cdots \cdots \\
& X_{N}^{q}=\pi^{h} X_{N}+\cdots+(-1)^{i}\left(\begin{array}{c}
h \\
i
\end{array}\right) \pi^{h-i} X_{N-i}+\cdots+(-1)^{h} X_{N-h}
\end{aligned}
$$

Because the action of $O$ on $G^{(h)}$ is transitive, $K\left(G^{(h)}\right)=K\left(X_{1}^{0}, \ldots, X_{N}^{0}\right)$, where $\left(X_{1}^{0}, \ldots, X_{N}^{0}\right) \in G^{(h)}$ is such that $X_{1}^{0} \neq 0$. As earlier, $\bar{\kappa}_{\alpha}$ establishes a one-to-one correspondence between $G^{(h)}$ and the set $G_{\alpha}^{(h)}$ of solutions $\left(X_{1 \alpha}^{0}, \ldots, X_{N \alpha}^{0}\right) \in K_{\text {sep }}^{N}$ of the system

$$
\begin{aligned}
& X_{1 \alpha}^{q}=\pi_{\alpha}^{h} X_{1 \alpha} \\
& X_{2 \alpha}^{q}=\pi_{\alpha}^{h} X_{2 \alpha}-\left(\begin{array}{c}
h \\
1
\end{array}\right) \pi_{\alpha}^{h-1} X_{1 \alpha}
\end{aligned}
$$

$$
X_{N \alpha}^{q}=\pi_{\alpha}^{h} X_{N \alpha}+\ldots+(-1)^{i}\left(\begin{array}{c}
h \\
i
\end{array}\right) \pi_{\alpha}^{h-i} X_{N-i, \alpha}+\ldots+(-1)^{h} X_{N-h, \alpha}
$$

(as earlier, $X_{i \alpha}=0$ if $\left.i<0\right)$. Choose $\left(X_{1 \alpha}^{0}, \ldots, X_{N \alpha}^{0}\right) \in G_{\alpha}^{(h)}$ such that $X_{1 \alpha}^{0} \neq 0$ and set $Z_{i}=X_{i}-X_{i \alpha}^{0 q^{M}}$. Notice that $K_{\alpha}\left(X_{1 \alpha}^{0}, \ldots, X_{N \alpha}^{0}\right)=\bar{\kappa}_{\alpha}\left(K\left(G^{(h)}\right)\right):=$ $K_{\alpha}\left(G_{\alpha}^{(h)}\right)$. Then $Z_{1}, \ldots, Z_{N}$ satisfy the following system of equations

$$
\begin{aligned}
& Z_{1}^{q}=\pi^{h} Z_{1}+F_{1} \\
& Z_{2}^{q}=\pi^{h} Z_{2}-\left(\begin{array}{c}
h \\
1
\end{array}\right) \pi^{h-1} Z_{1}+F_{2} \\
& \cdots \cdots \cdots \cdots \cdots \cdots \cdots \cdots \\
& Z_{N}^{q}=\pi^{h} Z_{N}+\ldots+(-1)^{i}\left(\begin{array}{c}
h \\
i
\end{array}\right) \pi^{h-i} Z_{N-i}+\ldots+(-1)^{h} Z_{N-h}+F_{N}
\end{aligned}
$$

where

$$
\begin{aligned}
& F_{1}=\widetilde{\pi^{h}} X_{1 \alpha}^{0 q^{M}} \\
& F_{2}=\widetilde{\pi^{h}} X_{2 \alpha}^{0 q^{M}}-\left(\begin{array}{c}
h \\
1
\end{array}\right) \widetilde{\pi^{h-1}} X_{1 \alpha}^{0 q^{M}} \\
& \ldots \ldots \ldots \ldots \ldots \ldots \ldots . . \\
& F_{N}=\widetilde{\pi^{h}} X_{N \alpha}^{0 q^{M}}+\ldots+(-1)^{i}\left(\begin{array}{c}
h \\
i
\end{array}\right) \widetilde{\pi^{h-i}} X_{N-i, \alpha}^{0 q^{M}}+\ldots+(-1)^{h-1} h \widetilde{\pi} X_{N-h+1, \alpha}^{0 q^{M}} .
\end{aligned}
$$


Set

$$
\delta(N, h)=\min \left\{v_{K}\left(X_{i \alpha}^{0 q^{M}}\right) \mid 1 \leqslant i \leqslant N\right\} .
$$

Clearly, $\delta(N, h)>0$. Notice that all $F_{i} \in K_{\alpha}\left(G_{\alpha}^{(h)}\right)$ and for $1 \leqslant i \leqslant N$, it holds

$$
v_{K}\left(F_{i}\right) \geqslant e(h+1-i)+\alpha^{*}+\delta(N, h)>e(N-i)+\frac{e h q}{q-1} .
$$

Now we can proceed with a small induction to find successively $Z_{1}$ then $Z_{2}$ etc. by using Lemma 11 for the first equation, then the second, etc. This will give the existence of a unique solution $\left(Z_{1}^{0}, \ldots, Z_{N}^{0}\right)$ of system $(7)$ such that for $1 \leqslant i \leqslant N, v_{K}\left(Z_{i}^{0}\right)>e\left(N-i+\frac{h}{q-1}\right)$. As earlier in n.3.3 this implies that $K\left(G^{(h)}\right) K_{\alpha}=K_{\alpha}\left(G_{\alpha}^{(h)}\right)$.

Proposition 12 is proved.

4.4. Now we are going to study the fields $K(g)$, where $g \in G$ is such that $X_{1}(g)=\cdots=X_{N}(g)=1$, cf. n.4.2.

Consider the following formal identity on the $K$-algebra $A$ of functions on $G$

$$
\left(\pi \operatorname{id}_{A}-[\pi]\right)^{-h}=\pi^{-h} \sum_{i \geqslant 0} \gamma_{i} \pi^{-i}[\pi]^{i}
$$

where for all $i \geqslant 0, \gamma_{i}=(-1)^{i}\left(\begin{array}{c}-h \\ i\end{array}\right)$. This allows us to rewrite system (5) in the following equivalent form:

$$
Y_{n}^{q}-\pi^{h} Y_{n}=-\sum_{1 \leqslant i<n} \frac{\gamma_{i}}{\pi^{i}} Y_{n-i}^{q}-u \sum_{0 \leqslant i<n} \frac{\gamma_{i}}{\pi^{i}}, 1 \leqslant n \leqslant N
$$

Use the notation and definitions from n.3.1.

Remind that for any $a \in K_{1}, \tilde{a}=a-\kappa_{\alpha}(a)^{q^{M}}$. If $a$ is given by a big formula then we shall use also the notation $\tilde{a}=(a)^{\sim}$. Until the end of this paper we assume that $\alpha=\frac{m}{q^{M}-1} \in \mathbb{Q}$ and $M \in \mathbb{N}$ are chosen such that

$$
\alpha\left(1-q^{-M}\right)=\alpha^{*}=e(N-1)+\frac{e q h}{q-1}-1-\varepsilon(\alpha),
$$

where

- $0<\varepsilon(\alpha)<q^{-N}$

- $\alpha q^{-M}<\varepsilon(\alpha)$ 
- $\varepsilon(\alpha)<\delta(N, h)$

(Use that rational numbers with zero $p$-adic valuation are dense in the set of all rational numbers and for a given $\alpha$ we can choose arbitrarily large $M)$. Notice that $\alpha^{*} q^{M}=m \in \mathbb{N}$ is prime to $p$ and this implies that the $p$-adic valuation of $\varepsilon(\alpha) q^{M}$ is zero. Notice also that under such a choice of $\alpha$ we can apply the result of proposition 12 .

As earlier, choose an extension $\bar{\kappa}_{\alpha}$ of $\kappa_{\alpha}$ to $K_{\text {sep }}$. Then it induces a one-to-one correspondence between the set of solutions of system (5) and the solutions of the system

(8) $Y_{n \alpha}^{q}-\pi_{\alpha}^{h} Y_{n \alpha}=-\sum_{1 \leqslant j \leqslant h}(-1)^{j-1}\left(\begin{array}{c}h \\ j\end{array}\right) \pi_{\alpha}^{h-j} Y_{n-j, \alpha}-u_{\alpha} \sum_{0 \leqslant j<n} \frac{\gamma_{i}}{\pi_{\alpha}^{i}}, \quad 1 \leqslant n \leqslant N$,

Fix a solution $g_{\alpha}=\left(Y_{1 \alpha}^{0}, \ldots, Y_{N \alpha}^{0}\right) \in K_{\text {sep }}^{N}$ of this system and set $K\left(g_{\alpha}\right)=$ $K\left(Y_{1 \alpha}^{0}, \ldots, Y_{N \alpha}^{0}\right)$. Introduce for $1 \leqslant n \leqslant N$, the new variables $Z_{n}$ such that $Z_{n}=Y_{n}-Y_{n \alpha}^{0 q^{M}}$.

Then $Z_{1}, \ldots, Z_{N}$ satisfy the following system of equations

(9) $Z_{n}^{q}-\pi^{h} Z_{n}=\widetilde{\pi^{h}} Y_{n \alpha}^{0 q^{M}}-\sum_{1 \leqslant i<n}\left(\frac{\gamma_{i}}{\pi^{i}}\right)^{\sim} Y_{n-i, \alpha}^{0 q^{M+1}}-\sum_{1 \leqslant i<n} \frac{\gamma_{i}}{\pi^{i}} Z_{n-i}^{q}-\sum_{0 \leqslant i<n}\left(\frac{\gamma_{i} u}{\pi^{i}}\right)^{\sim}$.

Proposition 13. System (9) has a solution $\left(Z_{1}^{0}, \ldots, Z_{N}^{0}\right)$ such that

a) if $1 \leqslant n<N$, then $Z_{n}^{0} \in K_{\alpha}\left(g_{\alpha}\right)$ and

$$
v_{K}\left(Z_{n}^{0}\right) \geqslant \frac{e h}{q-1}+e(N-n)-\varepsilon(\alpha)
$$

b) if $n=N$ then $Z_{N}^{0}-\gamma_{N-1} W^{0} \in K_{\alpha}\left(g_{\alpha}\right)$ where $W^{0}$ is a solution of the equation $W^{q}-\pi^{h} W=-\pi^{1-N} \tilde{u}$.

We shall prove this proposition below in $n .4 .5$ and use now this result to finish the proof of Theorem C.

Suppose $g \in G$ corresponds to the solution $\left(Y_{1 \alpha}^{0 q^{M}}+Z_{1}^{0}, \ldots, Y_{N \alpha}^{0 q^{M}}+Z_{N}^{0}, 1, \ldots, 1\right)$ of system (4). Then Proposition 13 implies that

$$
K(g) K_{\alpha}\left(g_{\alpha}\right)=K^{\prime} K_{\alpha}\left(g_{\alpha}\right),
$$


Characteristic p Analogue of Modules with Finite Crystalline Height 491

where $K^{\prime}=K_{\alpha}\left(W^{0}\right)$. Notice that

$$
v_{K_{\alpha}}\left(\pi^{-(N-1)} \tilde{u}\right)=q^{M} v_{K}\left(\pi^{-(N-1)} \tilde{u}\right)=\frac{e q^{M+1} h}{q-1}-q^{M} \varepsilon(\alpha)
$$

is a prime to $p$ integer. This implies easily that the maximal upper ramification number of $K^{\prime}$ over $K_{\alpha}$ is $v\left(K^{\prime} / K_{\alpha}\right)=q^{M} \varepsilon(\alpha)>\alpha$ and, therefore,

$v\left(K^{\prime} / K\right)=\max \left\{v\left(K_{\alpha} / K\right), \varphi_{K_{\alpha} / K}\left(q^{M} \varepsilon(\alpha)\right)\right\}=\alpha^{*}+\varepsilon(\alpha)=e\left(N-1+\frac{h q}{q-1}\right)-1$.

As $v\left(K^{\prime} / K\right)>\alpha$, Proposition 8 implies the equality $v(K(g) / K)=v\left(K^{\prime} / K\right)$, which proves Theorem $\mathrm{C}$.

\subsection{Proof of proposition 13.}

Lemma 14. Suppose $\left(Y_{1}^{0}, \ldots, Y_{N}^{0}\right) \in K_{\mathrm{sep}}^{N}$ is a solution of system (5). If $1 \leqslant$ $n \leqslant N$ and $r=r(n) \in \mathbb{Z}_{\geqslant 0}$ is such that $r h<n \leqslant(r+1) h$ then $v_{K}\left(Y_{n}^{0}\right)=q^{-r-1}$.

Proof. Use induction on $1 \leqslant n \leqslant N$.

If $n=1$ the statement is obviously true, because $Y_{1}^{0 q}-\pi^{h} Y_{1}^{0}=-u, v_{K}\left(Y_{1}^{0}\right)=$ $\frac{1}{q} v_{K}(u)$ and $r(1)=0$.

Suppose $n>1$ and the proposition is proved for all $n^{\prime}<n$.

If $n \leqslant h$ then $r(1)=\cdots=r(n)=0$ and for $1 \leqslant j<n$,

$$
v_{K}\left(\pi^{h-j} Y_{n-j}^{0}\right)=(h-j) e+\frac{1}{q}>1=v_{K}(u)
$$

and from the equation for $Y_{n}^{0}$ it follows $v_{K}\left(Y_{n}^{0}\right)=q^{-1}$.

If $n>h$ then $r(n) \geqslant 1$ and we have for $1 \leqslant j<h$,

$$
v_{K}\left(\pi^{h-j} Y_{n-j}^{0}\right) \geqslant e+q^{-r(n-1)-1}>1=v_{K}(u)>q^{-r(n-h)-1}=v_{K}\left(Y_{n-h}^{0}\right),
$$

Therefore, $v_{K}\left(Y_{n}^{0}\right)=\frac{1}{q} v_{K}\left(Y_{n-h}^{0}\right)=q^{-r(n-h)-2}=q^{-r(n)-1}$. The Lemma is proved.

Corollary 15. With the above notation for $1 \leqslant n \leqslant N, v_{K}\left(Y_{n \alpha}^{0 q^{M}}\right)=q^{-r(n)-1}$.

Lemma 16. Suppose $1 \leqslant n \leqslant N, 1 \leqslant i<n$. Then

a) $v_{K}\left(\widetilde{\pi^{-i}} Y_{n-i, \alpha}^{0 q^{M+1}}\right)>\frac{e q h}{q-1}+e(N-n)$ if $i \neq n-1$;

b) $v_{K}\left(\widetilde{\pi^{-i}} Y_{n-i, \alpha}^{0 q^{M+1}}\right) \geqslant \frac{e q h}{q-1}+e(N-n)-\varepsilon(\alpha)$ if $i=n-1$; 
c) $v_{K}\left(\widetilde{\pi^{h}} Y_{n \alpha}^{0 q^{M}}\right)>\frac{e h q}{q-1}+e(N-n)$;

d) $v_{K}\left(\frac{u}{\pi^{i}}\right)^{\sim} \geqslant \frac{e h q}{q-1}+e(N-n)-\varepsilon(\alpha)$.

Proof of lemma. We have

$$
v_{K}\left(\widetilde{\pi^{-i}} Y_{n-i, \alpha}^{0 q^{M+1}}\right) \geqslant \frac{e q h}{q-1}+e(N-n)+e(n-i-1)-1+q^{-r(n-i)}-\varepsilon(\alpha) .
$$

Then use that if $i \neq n-1$ then $e(n-i-1) \geqslant 1$ and if $i=n-1$ then $r(n-i)=0$. (Remind also that $\varepsilon(\alpha)<q^{-N}$.) This proves parts a) and b). Similarly,

$$
v_{K}\left(\widetilde{\pi^{h}} Y_{n \alpha}^{0 q^{M}}\right) \geqslant \frac{e h q}{q-1}+e(N-n)+h e-1-\varepsilon(\alpha)+q^{-r(n)-1} .
$$

Then c) follows because $h e \geqslant 1$ and $\varepsilon(\alpha)<q^{-N}$, cf. n.4.4. The part d) can be proved similarly.

The lemma is proved.

Now we can prove Proposition 13.

Use induction on $N$.

Suppose $N=1$. Then there is nothing to prove in a) and $Z_{1}^{0}$ appears as a solution of the equation

$$
Z_{1}^{q}-\pi^{h} Z_{1}=\widetilde{\pi^{h}} Y_{1 \alpha}^{0 q^{M}}-\gamma_{0} \tilde{u}
$$

Therefore, b) follows from Lemma 11 via the estimate c) of Lemma 16.

Now suppose $N>1$ and use induction on $1 \leqslant n<N$ to prove a). If $n=1$ then $Z_{1}^{0}$ must satisfy equation (10). Again use Lemma 11 by estimating the valuation of the right hand side of this equation via the part c) of Lemma 16.

Suppose $1<n \leqslant N$ and we have chosen the corresponding $Z_{1}^{0}, \ldots, Z_{n-1}^{0}$. Then for $1 \leqslant i<n$,

$$
\begin{gathered}
v_{K}\left(\pi^{-i} Z_{n-i}^{0 q}\right) \geqslant-i e+q\left(\frac{e h}{q-1}+e(N-n+i)-\varepsilon(\alpha)\right) \\
\geqslant \frac{e h q}{q-1}+e(N-n)+(q-1) e i-q \varepsilon(\alpha)>\frac{e h q}{q-1}+e(N-n),
\end{gathered}
$$

because $\varepsilon(\alpha)<q^{-N}<q^{-1}$, cf. n.4.4. 
This estimate together with estimates from Lemma 16 imply that the righthand side of equation (9) has $v_{K}$-valuation $\geqslant \frac{e h q}{q-1}+e(N-n)-\varepsilon(\alpha)$. Therefore, by Lemma 11 we can choose for $n<N$, the solution $Z_{n}^{0} \in K_{\alpha}\left(g_{\alpha}\right)$ with

$$
v_{K}\left(Z_{n}^{0}\right) \geqslant e h /(q-1)+e(N-n)-\varepsilon(\alpha) .
$$

This proves the part a).

If $n=N$ then for a similar reason, the right-hand side of (9) can be written as

$$
-\gamma_{N-1}\left(\left(\pi^{1-N}\right)^{\sim} Y_{1 \alpha}^{0 q^{M+1}}+\left(\pi^{1-N} u\right)^{\sim}\right)+a,
$$

where $a \in K_{\alpha}\left(g_{\alpha}\right)$ and $v_{K}(a)>e h q /(q-1)$.

Then notice that:

1) $Y_{1 \alpha}^{q^{M+1}}=\pi_{\alpha}^{h q^{M}} Y_{1 \alpha}^{q^{M}}-u_{\alpha}^{q^{M}}$

2) the $v_{K}$-valuation of $\left(\pi^{1-N}\right)^{\sim} \pi_{\alpha}^{h q^{M}} Y_{1 \alpha}^{0 q^{M}}$ is strictly bigger than $e h q /(q-1)$;

3) there is an identity $\left(\frac{u}{\pi^{N-1}}\right)^{\sim}-\left(\frac{1}{\pi^{N-1}}\right)^{\sim} u_{\alpha}^{q^{M}}=\frac{1}{\pi^{N-1}} \tilde{u}$.

This implies that the right-hand side of (9) in the case $n=N$ is equal to $-\frac{\gamma_{N-1}}{\pi^{N-1}} \tilde{u}+a^{\prime}$, where $a^{\prime} \in K_{\alpha}\left(g_{\alpha}\right)$ and $v_{K}\left(a^{\prime}\right)>e h q /(q-1)$.

Therefore, by Lemma 11 the part b) is proved.

\section{REFERENCES}

[Ab1] V.Abrashkin, Group schemes of period p over the ring of Witt vectors. (Russian), Dokl. Akad. Nauk SSSR 283 (1985), no. 6, 1289-1294.

[Ab2] V.Abrashkin, Ramification in etale cohomology, Invent. Math. 101, (1990), no. 3, 631640.

[Ab3] V.Abrashkin, Modular representations of the Galois group of a local field and a generalization of a conjecture of Shafarevich, Izv. Akad. Nauk SSSR Ser. Mat. 53(1989), no. 6, 1135-1182; Engl. transl. in, Math. USSR-Izv. 35(1990), no. 3, 469-518.

[Ab4] V.Abrashkin, The ramification filtration of the Galois group of a local field. III, Izv. Ross. Akad. Nauk Ser. Mat. 62(1998), no. 5, 3-48 ; Engl. transl. in, Izv. Math. 62 no. 5 (1998), 857-900.

[Ab5] V.Abrashkin, Towards explicit description of ramification filtration in the 2-dimensional case, J. Théor. Nombres Bordeaux, 16(2004), no. 2, 293-333.

[Ab6] V.Abrashkin, Galois modules arising from Faltings's strict modules, Compositio Math. 142(2006), 867-888.

[An] G.Anderson, t-motives, Duke Math. J. 53(1986), no. 2, 457-502. 
[Fa] G.Faltings, Group schemes with strict $\mathcal{O}$-action, Moscow Math. J. 2(2002), no. 2, 249279.

[Fo1] J.-M.Fontaine, Il n'y a pas de variété abélienne sur $\mathbb{Z}$, Inv. Math. 81(1985), no. 3, 515538 .

[Fo2] J.-M.Fontaine, Schémas propres et lisses sur $\mathbb{Z}$, Proc. of Indo-French Conference on Geometry (Bombay, 1989), Hindustan book agency, Delhi, 1993, p. 43-56.

[Fo3] J.-M.Fontaine, Représentations p-adiques des corps locaux.I., The Grothendieck Festschrift, Progr. Math., 87, Birkhäuser Boston, Boston, MA, 1990, vol. II, p. 249309.

[Ga] P. Gabriel, Étude infinitésimale des schémas en groupes, Schémas en Groupes I, Lecture notes in Mathematics (Springer-Verlag, eds.), vol. 151, 1970, p. 474-560.

[Sc] R. Schoof, Abelian varieties over $\mathbb{Q}$ with bad reduction in one prime only, Compos. Math. 141 (2005), no. 4, 847-868.

[Se] J.-P.Serre, Corps locaux, Deuxième édition. Publication de l'Université de Nancago, No. VIII. Hermann, Paris, 1968.

Victor Abrashkin

Maths Dept., Durham University

Sci. Laboratories, South Rd., Durham, DH1 3LE, U.K.

E-mail: victor.abrashkin@durham.ac.uk 